\title{
The Fabry-Pérot interferometer prototype for the ADAHELI solar small mission
}

\author{
Francesco Berrilli ${ }^{a}$, Martina Cocciolo ${ }^{a}$, Luca Giovannelli ${ }^{a}$, Dario Del Moro $^{a}$, Fabio \\ Giannattasio $^{a}$, Roberto Piazzesi ${ }^{a}$, Marco Stangalini $^{a}$, Alberto Egidi ${ }^{a}$, Fabio Cavallini $^{b}$, \\ Vincenzo Greco $^{c}$ and Stefano Selci ${ }^{d}$ \\ ${ }^{a}$ Dipartimento di Fisica, Universitá di Roma Tor Vergata, Via Ricerca Scientifica, 00133 \\ Roma, Italy \\ ${ }^{b}$ INAF - Osservatorio Astrofisico di Arcetri, Largo Enrico Fermi 6, 50125 Firenze, Italy \\ ${ }^{c}$ Istituto Nazionale di Ottica Applicata - CNR, Largo Enrico Fermi 6, 50125 Firenze, Italy \\ ${ }^{d}$ Institute for Complex Systems - CNR, Via Fosso del Cavaliere, 100 00133, Rome, Italy
}

\begin{abstract}
ADAHELI ADvanced Astronomy for HELIophysics is a solar satellite designed to investigate the fast dynamics of the solar photosphere and chromosphere performing visible and NIR broad-band and monochromatic observations of selected atomic lines. ADAHELI is an Italian Space Agency (ASI) project, approved for a feasibility study within the ASI Small Missions call. ISODY Interferometer for SOlar DYnamics is a Gregorian telescope and its focal plane suite (FPS). The FPS is composed of a high-resolution fast acquisition system, based upon a tandem of Fabry-Pérot interferometers operating in the visible and NIR regions on selected solar atmospheric lines, a broad band channel, and a correlation tracker used as image stabilization system. In this contribution we describe the Fabry-Pérot étalon prototype, based on the capacitance-stabilised concept, realized in our laboratory to perform preliminary mechanical and optical tests with a view to a future Fabry-Pérot étalon prototype for space application.
\end{abstract}

Keywords: Fabry-Pérot Interferometer, space telescopes, spectroscopy, solar physics

\section{INTRODUCTION}

High-cadence and high-resolution spectro-polarimetric observations of the lower solar atmosphere are a key tool to investigate highly dynamic phenomena present in these layers of the atmosphere of our star. A fast camera system and a panoramic and high-transparent spectrometer allow us to obtain a suitable cadence to study highfrequency oscillations and fast-moving plasma present in the solar photosphere and chromosphere.

The spatial resolution depends primarily on the diameter of the telescope entrance pupil and on the observed wavelengths. However, as far as the spectrometer is concerned, to completely exploit the ADAHELI telescope resolution, two conditions must be satisfied: the instrument itself must not impair the optical quality of the telescope, and the detector must allow a suitable spatial sampling. Moreover, a high spectral resolving power $(R \geq 200000)$ is required to properly analyze narrow photospheric lines and a high temporal resolution (several frames per second) is necessary to investigate highly dynamic solar phenomena. The exposure time must be sufficiently short to satisfy the Nyquist frequency associated with the analysis techniques required to exploit the acquired dataset. A sufficiently large field of view (FOV) is essential to easily study active regions and a suitably extended wavelength range, visible-NIR, is needed to offer a broad option among lines with different diagnostic power. Finally, a high wavelength stability (maximum drift $0.02 \mathrm{pm}$ in 10 hours) is mandatory to provide a good reproducibility of the selected spectral points in long observing runs (e.g., oscillatory phenomena).

To this purpose, the Fabry-Pérot interferometer (FPI) seems to be a good candidate. Actually, thanks to its large area and to the low absorption coefficients coatings this device fulfills the required characteristics. Moreover,

Further author information:

Berrilli, F.: E-mail: berrilli@roma2.infn.it, Telephone: +39 0672594430 


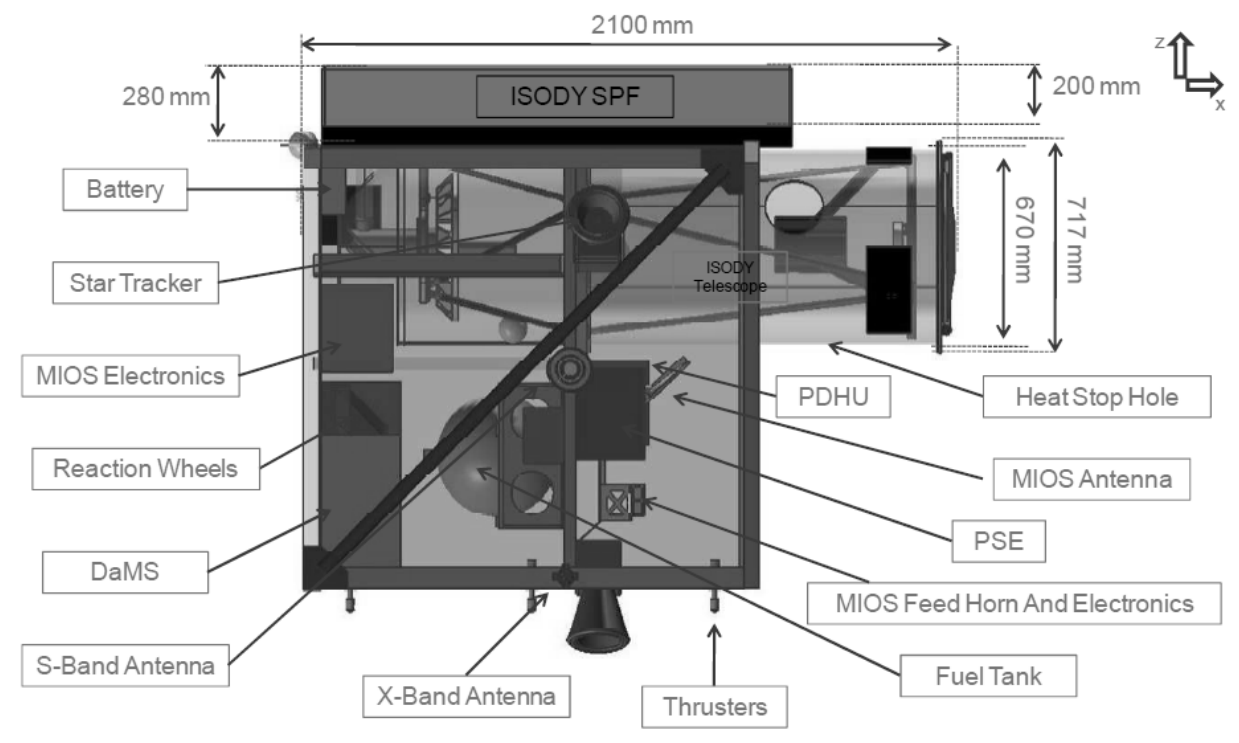

Figure 1: ADAHELI Configuration

FPIs have high achievable spectral resolution, rapid wavelength tuning, if piezo-scanned, and high stability, if capacitance-stabilised and thermo-stabilized.

A short description of the ADAHELI mission is reported in Section 2, while in Section 3 a description of ISODY, the Gregorian telescope and focal plane suite operating in the visible-NIR spectral range is presented. In Section 4 criticalities of space Fabry-Pérot interferometers (FPI) for ISODY are presented, followed by a description of the design of the FPI prototype in Section 5. Finally, concluding remarks and a short description of a second FPI prototype of the instrument are given in Section 6.

\section{THE ADAHELI MISSION}

ADAHELI is a small-class low-budget satellite mission for the study of the solar photosphere and the chromosphere and for monitoring solar flare emission. ADAHELI's design has completed its Phase-A feasibility study in December 2008, in the framework of ASI (Agenzia Spaziale Italiana) 2007 Small Missions Program. GGS SpA was leader industry and University of Rome Tor Vergata was leader scientific institution of the project.

During its Nominal Mission (two plus one years) ADAHELI shall constantly point the Sun, except during manouvres, eclipses or contingencies. The spacecraft radial velocity in the sunward direction, shall not exceed $\mp 4 \mathrm{~km} / \mathrm{s}$, during $95 \%$ of the yearly orbit. The initial accuracy in pointing a selected Region Of Interest (ROI) must be a small fraction of the field of view, say $<10$ arcsec, to get the ROI within the field of view of the high resolution ISODY telescope. The precision in tracking the ROI must be significantly better, i.e. $<0.1$ arcsec for the whole duration of the acquisition, to allow the planned high quality of the image series. This must be achieved by the combined action of the satellite Attitude and Orbit Control Subsystem (AOCS) and of the correlation tracker correction system inside the telescope. The satellite relative velocity with respect to the Sun center shall be known within $1 \mathrm{~cm} / \mathrm{s}$. These very challenging requirements, flow down constrain the design of the main instrument, ISODY. Further details on the mission and its requirements may be found in 1,2.

The satellite configuration is characterized by a prismatic bus with body fixed solar array and payloads mounted as shown in Fig.1. The proposed configuration is compatible with the VEGA launcher.

\section{THE FOCAL PLANE ASSEMBLY OF THE ISODY INSTRUMENT}

ISODY is designed to obtain high resolution spatial, spectral, and temporal polarimetric images of the solar photosphere and chromosphere. The Focal Plane Assembly of the ISODY instrument comprises two visiblenear-infrared science optical paths or channels: the Narrow Band (NB) and the Broad Band (BB) channels, 


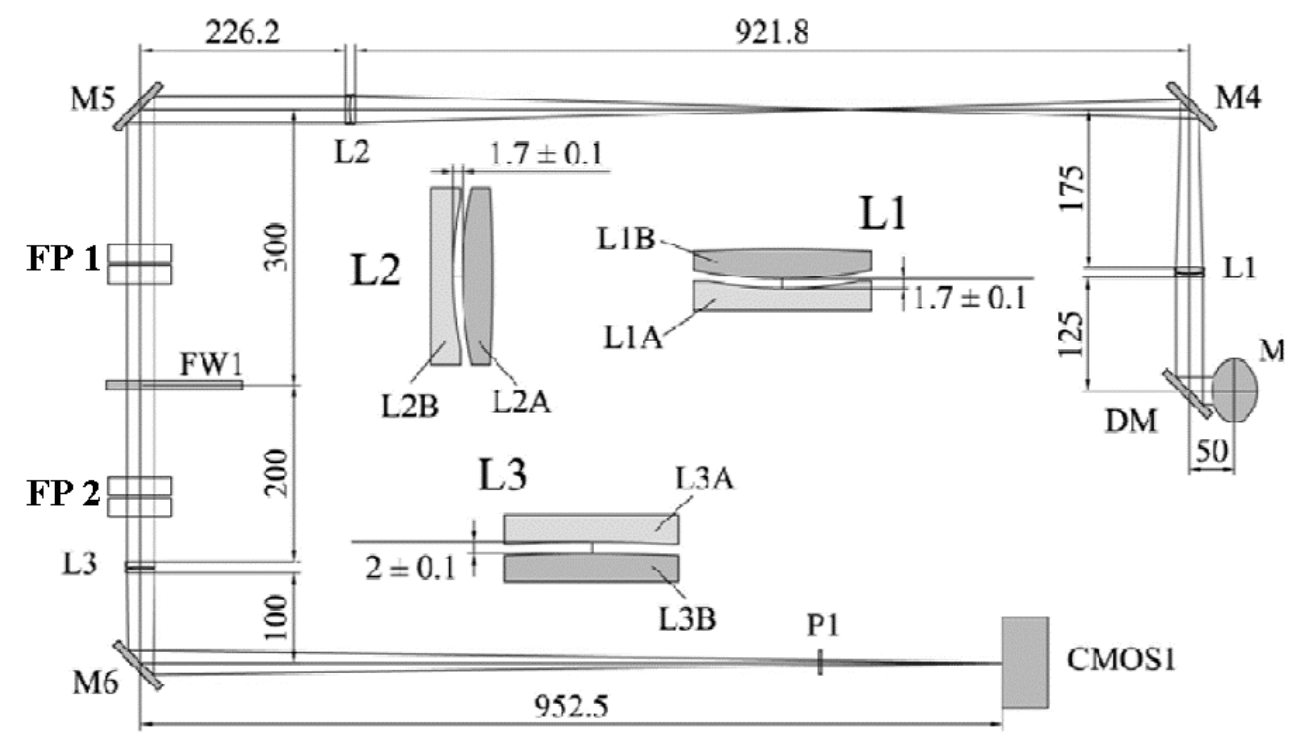

Figure 2: The optical path and the relative positions of the optical elements/units of ISODIY Narrow Band channel.

as well as the Correlation Tracker (CRTR) channel. The optical path with the relative positions of the optical elements/units for the NB channel is shown in Fig. 2 and consist in 25 items and/or assemblies, as briefly described in this paragraph. A dichroic mirror (item DM on the figure) transmits part of the telescope beam towards the $\mathrm{BB}$ channel in the wavelength range $530-670 \mathrm{~nm}$ and reflects part of the beam to the NB channel in the range 850-860 nm. The principal optical path of the NB channel is formed by three folding mirrors (M4, M5 and M6) and three converging lenses (L1, L2 and L3), which successively collimate the solar and the pupil images. After L2, there are two Fabry-Pérot interferometers (FP1, FP2) used in axial-mode and in classic mount and, between them, a filter wheel (FW) carrying a hole, a dark slide and four interference filters.

The principal optical path of the BB channel, simpler than the NB channel, is formed by two small folding mirrors, a re-imaging lens unit, a variable neutral density and a filter wheel, carrying a hole, a dark slide and four interference filters. A variable neutral density adjusts the radiation flux for different wavelengths and different exposure times. The optical path of the CRTR channel is common to the Broad Band channel up to a beam splitter, which provides a separate CRTR channel. All the optical instrumentation is mounted on an Optical Bench of honeycomb with sufficient stiffness to be self-supporting and maintain a good planarity on-ground and in-orbit. The envelope of the complete Focal Plane Assembly is 1600 x 900 x $280 \mathrm{~mm}$. The instrumentation is enclosed in a box of dimensions 1600 x 900 x $200 \mathrm{~mm}$.

Narrow Band specifications of the two Fabry-Pérot interferometers are summarized in Table 1. A Classical Mount (CM) has been adopted for the system. From the spectroscopic point of view this mount, with respect $\mathrm{t} t$ to the telecentric one, has the advantage of a transparency profile with the same shape at all the points of the final image. Moreover, its systematic blue-shift is not difficult to correct, allowing use of larger incidence angles than the telecentric mounting, which needs, on the contrary, small relative apertures to achieve good image quality and spectral resolution. This implies that the CM generally allows a larger FOV. ${ }^{3}$

Further details on the ISODY instrument may be found in 4 . 
Table 1: Narrow Band specifications of the two Fabry-Pérot interferometers.

\begin{tabular}{|l|l|}
\hline Plate parallelism and spacing & Capacity servo-controlled \\
\hline Diameter $(\mathrm{mm})$ & 50 \\
\hline Spacing $(\mathrm{mm})$ & $2.290,0.635$ \\
\hline Reflectivity $(\%)$ & 93 \\
\hline Absorption coefficient & $\leq 0.002$ \\
\hline Large scale defects & $\leq \lambda / 100(\lambda=632.8 \mathrm{~nm})$ \\
\hline Small scale defects $(\mathrm{nm})$ & Gaussian $\sigma \leq 1$ \\
\hline
\end{tabular}

\section{CRITICALITIES OF SPACE FABRY-PÉROT ÉTALON}

The Fabry-Pérot etalon is the basis of most types of medium- to narrow-bandwidth filters and is available in many different configurations. It consists of two transparent plates whose facing surfaces are optically flat and coated with a semi-reflective dielectric coating. The Fabry-Pérot plates are separated by spacers, creating a well-defined optical cavity between the coated surfaces (known as the Optical Path Difference or OPD). The etalon or cavity acts as an optical resonator, promoting interference between the beams of the reflected light within the cavity, depending on the optical path difference. In this way, the etalon allows transmission of light at well-defined wavelengths and angles of incidence to the cavity. ${ }^{5}$ The development of narrow-bandwidth optical filter technology for use in space applications requires special qualification levels of temperature cycle/shock, thermal/vacuum and vibration/shock tests. ${ }^{6}$

\subsection{Mechanical stability}

The two ISODY Fabry-Pérot etalons are controlled and stabilised with a capacitive servo control system of plate parallelism and separation. Each FPI is mechanically mounted in such a way that the opposite plates are parallel (this can be easily done with the precision of a few arcseconds). However, the launch stresses can introduce an unwanted tilt producing a wavelength shift of the transparency profile of a FPI with respect to the other. This shift is zero along the axis of tilt. Instead, it grows larger toward the edge of the field of view. The final effect is a detuning between the two interferometers which produces a deformation of the instrument profile and an uneven obscuration. In Fig. 3 the effects due to a tilt between the two FPI is shown. Tilt values equal to $0.1^{\circ}$, with different sign for the two FPIs, have been adopted to best visualize the detuning effects.

In order to maintain the transparency variation within $1 \%$ the maximum allowable tilt angle between the two FPIs cannot exceed \pm 30 arcsec.

\subsection{Thermal stability}

Changes in length of the piezoelectric actuators, due to temperature variations, are sensed by the electronic control which reacts by changing the Fabry-Pérot cavity OPD. This implies that the OPD, and therefore the position in wavelength of the profile of transparency, is sensitive to temperature. We have two special cases: 1 - The temperature varies in the same way for both Fabry-Pérot etalons. In this case, if $K$ is the thermal expansion coefficient of the actuator, we have:

$$
\frac{\Delta \lambda}{\lambda}=\frac{\Delta T}{T}=K
$$

since:

$$
\frac{\Delta \lambda}{\lambda}=\frac{v}{c}
$$

Corresponding to a spurious Doppler speed signal: 

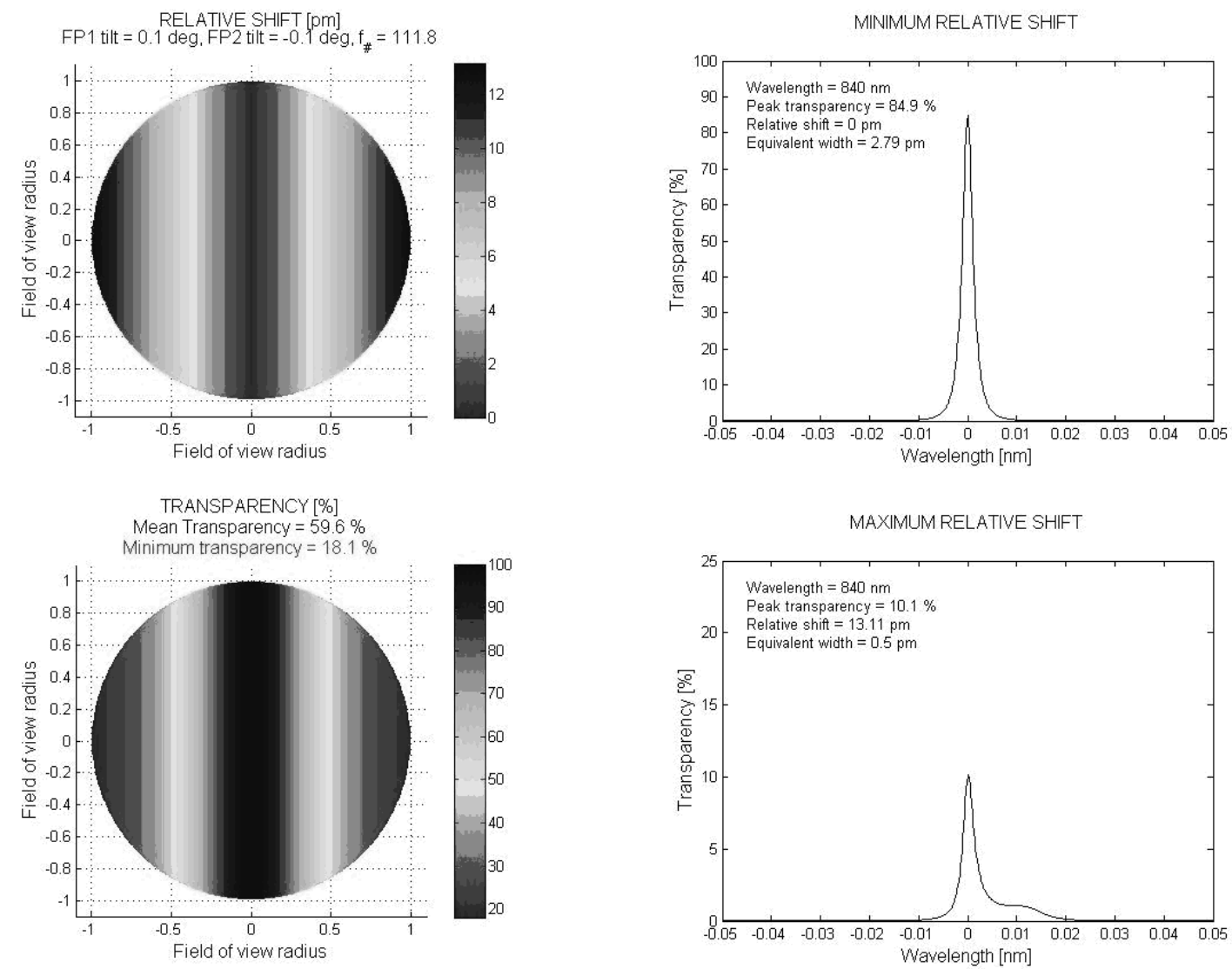

Figure 3: Tilt produces a shift in wavelength of the transparency profile. The shift is zero along the axis of tilt, while, in the direction perpendicular to this, it grows toward the edge of the field of view. The effect is a detuning between the two interferometers, which produces a deformation of the profile instrument and an uneven darkening. 


$$
v=K \times c=45 m s^{-1} C^{-1}
$$

The temperature of the two interferometers, which would not necessarely be the same, has to be kept constant within $\pm 0.5{ }^{\circ} \mathrm{C}$ to have a maximum drift of the instrumental profile of $\pm 25 \mathrm{~ms}^{-1}$. For the calculation we considered actuators made of Zerodur $\left(K=0.15 \times 10^{-6}{ }^{\circ} C^{-1}\right)$.

2 - The temperature varies differently for the two FP. In this case the transparency profiles of the two interferometers undergo different wavelength shifts that cause a detuning, a deformation of the instrumental profile and

a loss of transparency. In order to keep the transparency reduction of the instrumental below 1\%, we have to keep the temperature difference the two FPIs within $5^{\circ} \mathrm{C}$.

\section{FABRY-PÉROT INTERFEROMETER PROTOTYPE: DESIGN CONCEPTS}

\subsection{Introduction}

In order to test the reliability of the proposed fine adjustment system, a prototype of the interferometer has been designed for a laboratory test. This prototype shares similar optical and control system components with the final FPI for space application.

The proposed control system for the test prototype allows two levels of adjustment: a coarse one, controlled by micrometers, and a fine one, controlled by piezoelectric actuators. Position errors are measured by high sensitivity capacitive sensors. The double adjustment has been introduced to extend the working range of wavelengths and to compensate possible mechanical misalignments in the test phase. The double adjustment requires a complex optomechanical structure which has been considered only for the test prototype. The Fabry-Pérot etalon for space application will have a reduced spectral adjustment range.

\subsection{Hardware}

The optomechanics of the laboratory prototype has been designed to house: a 1 inch $(2.54 \mathrm{~cm})$ optical cavity, three micrometers, three piezoelectric actuators, three high sensitivity capacitive sensors. The adopted optomechanics has to control and guarantee the parallelism of the etalon optical surfaces during the wavelength scan, within the optical tolerances defined above.

The etalon main dimensions are: external diameter $=2.54 \mathrm{~cm}$, thickness of the optical surfaces $=15 \mathrm{~mm}$. Maximum displacements are $12 \mathrm{~mm}$ for micrometers and $15 \mu \mathrm{m}$ for piezoelectric actuators. The high sensitivity capacitive sensors working distance is $50 \mu \mathrm{m}$.

The control loop is managed by a dedicated controller, calibrated on the selected piezoelectric actuators and capacitive sensors.

\subsection{Requirements}

The definition of the optical requirements has already been discussed. Optical parameters constrain the distance between the cavity surfaces and the tolerances on their parallelism, specifying the minimum displacement that has to be controlled by the fine adjustment.

Operating requirements concern the survival of the components and their correct functioning in the operating environment. The test ground-based Fabry-Pérot will be subject to gravity loads; the space Fabry-Pérot interferometer instead will undergo temperature cycle/shock, thermal/vacuum and vibration/shock and severe accelerations during the satellite launch, and will operate in vacuum.

In the case of the prototype interferometer, optical requirements set the operating distance between the etalon surfaces to $10 \mathrm{~mm}$. Using the coarse adjustment this distance can be changed, as the micrometers allow displacements of the order of millimeters, broadening the range of available spectral lines. Main operating requirements concern the minimization of misalignments of the optical surfaces due to gravity load, and minimization of hysteresis at contact areas.

For the space interferometer the operating requirements involve the adoption of a different design, based on a one-piece concept to minimize the number of mechanical parts, the possible different displacements due to thermal loads and to keep the optical system aligned. 


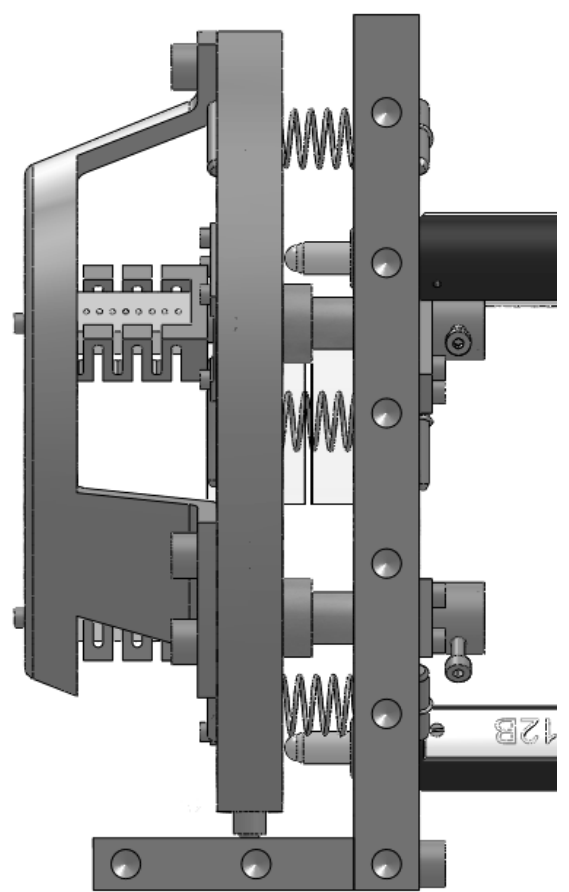

Figure 4: Detail of the test prototype optomechanical design.

\subsection{Prototype design}

The design of the test prototype is based on two concepts: ease of manufacture and modularity. The main aim of the optomechanics is, apart from compensating gravity deformations, the decoupling of coarse and fine adjustments. Each optical surface is housed in a different component: one of them is fixed to a base plate, the other is housed in a mobile plate which rests on the base plate by the use of a ball-tip screw. The ball-tip screw is coupled with a $\mathrm{V}$-groove in order to allow rotations and the displacement parallel to the optical axis. Adjustments are performed by three micrometers and three piezoelectric actuators placed in a $120^{\circ}$-symmetry around the etalon. The feedback for the control system is guaranteed by the high sensitivity capacitive sensors, placed in a $120^{\circ}$-symmetry on the backside of the piezoelectric actuators. The control system involves the use of a simple program which inputs the scan sequence during the observation, and the use of the loop controller to manage the fine adjustment by the piezoelectric actuators based on the capacitive sensors measurements. Fig.4 shows a detailed view of the test prototype optomechanical design. In order to realize kinematic couplings, both micrometers and piezoelectric actuators are equipped with ball-tip ends acting on a V-groove. Both the contact surfaces will be made in stainless steel to reduce hysteresis in the contact area. Decoupling of the coarse and fine adjustments is achieved by separating the mobile plate into two concentric rings. Micrometers act on the outer ring, the contact being preserved by the use of three preloaded springs in $120^{\circ}$-symmetry; the inner ring, which houses the mobile optical surface, is controlled by the piezoelectric actuators; the outer ring is connected to the inner ring by three V-shaped flexures. Piezoelectric actuators are kept in place by the use of a third support plate, attached to the outer ring of the mobile plate. Thus, a micrometer displacement moves both the mobile and the support plates, while a piezoelectric actuator displacement will move just the inner ring of the mobile plate. The left panel of Fig.5 shows the realization of the decoupling. The right panel of Fig.5 shows the complete assembly of the prototype optomechanics.

Etalon optical surfaces are connected to their relative plate by means of three equally spaced contact areas placed along each cylinder side surface. Each contact area is connected to its plate by a flexure device. A dedicated tool has been studied to assemble precisely the optical surface to its three flexure devices.

Many configurations have been studied, using different materials, flexures shapes and retaining springs rigidity. Optical requirements set the maximum displacement allowed at $50 \mathrm{~nm}$ along the optical axis. The material 

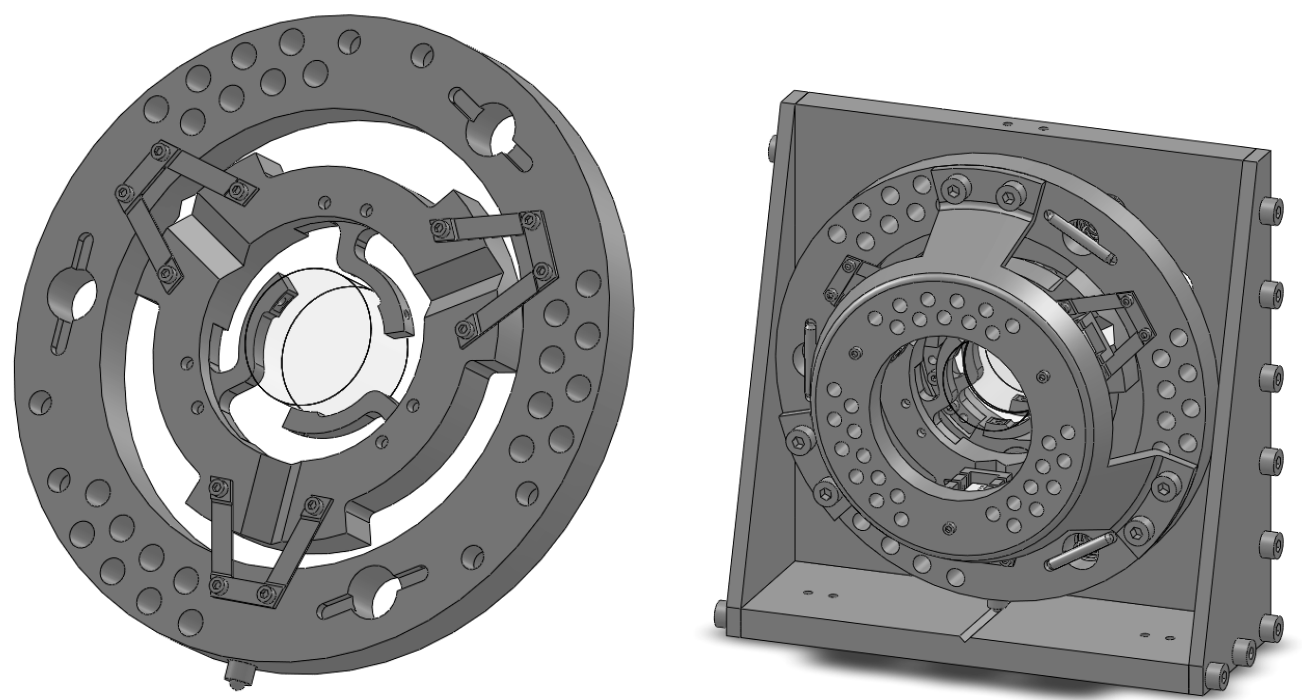

Figure 5: Left panel: Decoupling mechanism for coarse and fine adjustments. Right panel: dimetric view of the test prototype optomechanics.

selected for the optomechanical hardware is stainless steel. The main features that have been considered were: a) material cost and availability, b) elastic modulus, c) surface hardness, d) electrical conductivity, e) coupling with off-the-shelf components (screws, springs).

Analyses were performed in two loading conditions: gravity load and actuator displacement. Given the interferometer layout, gravity acts in the direction normal to the base plate, generating unwanted distortion in every operating condition. Unpredicted deflections of the fixed optical surface have to be avoided, for its position cannot be measured during the observations.

With regard to actuators displacements, two typical conditions have been analyzed: frequency scan (or shift, referring to coarse adjustment) and parallelism adjustment. The former load case involves the actuators performing a synchronized correction. The latter involves a change in the mobile optical surface inclination, that is to say a differential actuator displacement to obtain the necessary inclination angle of the optical surface.

Optomechanical hardware rigidity resulted as extremely important in the test prototype design. All the components should have a rigid body behavior during the adjustment operations, because the capacitive sensors measurement is taken on the inner ring of the mobile plate (not on the mobile optical surface directly). Furthermore, displacement of the fixed optical surface should be avoided because it cannot be measured directly during observations. At the same time, another important point is the correct design of the flexures connecting the inner and outer rings of the mobile plate. The aim of these flexures is double: $i$ to keep the inner ring in place against the gravity action, $i i$ to create a weak constraint, avoiding interference with the fine adjustment. Typical and maximum displacements conditions have been studied, both in frequency scan and parallelism adjustments.Results showed that the effect of gravity load on the etalon surfaces is acceptable. The maximum displacement value obtained on the mobile optical surface was about $95 \mathrm{~nm}$ in the direction of the gravity load.

\subsection{Space interferometer design}

The design of the interferometer for space implementation is different with respect to the test prototype. The more demanding operating environment requires a stiffer structure able to keep the alignment during the satellite launch and to compensate for the high thermal loads.

The ADAHELI mission requires the adoption of a double Fabry-Pérot interfermeter with 2 inche etalons having different spacings: the former using $2.29 \mathrm{~mm}$, the latter $635 \mu \mathrm{m}$. Satellite specifications set the maximum optomechanical hardware volume to $80 \times 80 \times 60 \mathrm{~mm}$ ( $80 \mathrm{~mm}$ maximum diameter) for both the interferometers, and the operating and survival temperatures, respectively $+5^{\circ} \mathrm{C} \backslash+30^{\circ} \mathrm{C}$ and $-20^{\circ} \mathrm{C} \backslash+80^{\circ} \mathrm{C}$. The mass of each Fabry-Pérot interferometer cannot exceed 520g. The double adjustment mechanism, involving the possibility to 
extend the operating wavelenght, cannot be used because of the extremely reduced operating distance of the capacitive sensors and the use of compliant flexures and springs. Mechanical vibrations and high thermal loads are sources of misalignments that have to be compensated during the different stages of the mission. Moreover manufacturing processes might introduce other potential sources of misalignments.

In order to minimize these effects, a good solution is to house both the etalon surfaces in a one-piece optomechanical structure. Starting from a cylindrical blank, proper machining can be obtain two rings (one for each etalon) connected by three elastic elements. The monolithic structure avoids connections between separate parts that experience mechanical vibrations, and is completely made of the one material, which removes thermal expansion coefficient differences. Piezoelectric actuators can be housed in this monolithic structure directly, making the interferometer design compact and reliable.

The chosen material is Invar, a nickel-steel alloy having an extremely low thermal expansion coefficient. Etalons, made of fused silica, will be connected to the optomechanical hardware using low outgassing adhesive which can operate in a vacuum environment.

Special care has to be paid to decouple vibrations induced by the spacecraft and the interferometer natural frequencies; moreover, in order to reduce the amplitude of the vibrations which can damage both etalons and piezoelectric actuators, a suitable damping device will be studied.

\section{CONCLUSIONS}

In this paper, we presented the design of a laboratory prototype Capacitance Stabilized Fabry-Pérot etalon. The manufacture of all the components is nearly complete. We plan to start the assembly in September 2011 and testing is due to start before the end of 2011. We plan to make a prototype space implementation of the present interferometer in order to start space qualification tests.

\section{ACKNOWLEDGMENTS}

This work was supported by Italian Space Agency (ASI) Phase A Contract I/020/08/0 ADAHELI. We thank Marco Velli, Luca Rosselli, Alberto Bigazzi, Paolo Sabatini and the ADAHELI Team for their valuable help in supporting the ADAHELI project.

\section{REFERENCES}

[1] Berrilli, F., Velli, M., Roselli, L., Bigazzi, A., and the ADAHELI Team, "The adaheli (advanced astronomy for heliophysics) solar mission," Mem. SAIt 80, 251 (2009).

[2] Berrilli, F., Bigazzi, A., Roselli, L., Sabatini, P., Velli, M., Alimenti, F., Cavallini, F., Greco, V., Moretti, P., Orsini, S., Romoli, M., White, S. M., and Team, A., "The adaheli solar mission: Investigating the structure of sun's lower atmosphere," Advances in Space Research 45, 1191-1202 (2010).

[3] Cavallini, F., "Ibis: A new post-focus instrument for solar imaging spectroscopy," Solar Physics 236, 415 (2006).

[4] Greco, V., Cavallini, F., and Berrilli, F., "The telescope and the double fabry-perot interferometer for the adaheli solar space mission," in [Space Telescopes and Instrumentation 2010: Optical, Infrared, and Millimeter Wave], Oschmann, J., Clampin, M., and MacEwen, H., eds., Proc. SPIE 7731, 773142-773142-9 (2010).

[5] Born, M. and Wolf, E., [Principles of Optics], Cambridge University Press (2002 (seventh edition)).

[6] Bond, R., Foster, M., Thompson, V.K., R. D., and Do Carmo, J. P., "High-resolution optical filtering technology," in [22nd Internation Laser Radar Conference (ILRC 2004)], ESA SP 56, 239 (2004). 